\title{
Calcofluor White Stain
}

National Cancer Institute

\section{Source}

National Cancer Institute. Calcofluor White Stain. NCI Thesaurus. Code C85880.

A fluorescent stain that binds to the cell wall. It is used for the detection of fungi, parasites, and yeasts, using fluorescent microscopy. 